CERN-PH-TH/2007-010

\title{
Successful Yukawa structures in Warped Extra Dimensions
}

\author{
J. I. Silva-Marcos 1 \\ CFTP, Departamento de Física \\ Instituto Superior Técnico, Avenida Rovisco Pais, 1 \\ 1049-001 Lisboa, Portugal
}

\begin{abstract}
For a RS model, with SM fields in the bulk and the Higgs boson on the TeV-brane, we suggest two specific structures for the Yukawa couplings, one based on a permutation symmetry and the other on the Universal Strength of Yukawa couplings hypothesis (USY). In USY, all Yukawa couplings have equal strength and the difference in the Yukawa structure lies in some complex phase. In both scenarios, all Yukawa couplings are of the same order of magnitude. Thus, the main features of the fermion hierarchies are explained through the RS geometrical mechanism, and not because some Yukawa coupling is extremely small. We find that the RS model is particularly appropriate to incorporate the suggested Yukawa configurations. Indeed, the RS geometrical mechanism of fermion locations along the extra dimension, combined with the two Yukawa scenarios, reproduces all the present experimental data on fermion masses and mixing angles. It is quite remarkable that in the USY case, only two complex phases of definite value $\pm \frac{\pi}{2}$ are sufficient to generate the known neutrino mass differences, while at same time, permitting large leptonic mixing in agreement with experiment.
\end{abstract}

PACS numbers: 11.10.Kk, 12.15.Ff, 14.60.Pq, 14.60.St

${ }^{1} \mathrm{e}-\mathrm{mail}:$ juca@cftp.ist.utl.pt 


\section{Introduction}

Lately, there has been great interest in extra dimension models. Extra dimension models are inspired by string theory, which itself is based on the existence of additional spatial dimensions [1]. As known, string theory 2 is a main candidate for an all-including quantum theory which allows for gravity, thus unifying all elementary particle interactions.

There exist several models of extra dimensions: universal extra dimension models in which all Standard Model (SM) fields may propagate in extra dimensions [3, brane universe models in which the SM fields live in our 3-dimensional spatial subspace [4, 5, or intermediate models in which only gauge bosons and the Higgs fields propagate in extra dimensions while fermions are 'stuck' at fixed points along these dimensions [6]. Amongst the brane universe models two different scenarios have attracted much attention: one suggested by Arkani-Hamed, Dimopoulos and Dvali (ADD) with large flat extra dimensions [7, and the other by Randall and Sundrum (RS) with a single small warped extra dimension $[8,9] 2$.

Extra dimensional models have some advantages over supersymmetric theories [10]. Besides the fact that they lead to the unification of the gauge couplings, either at high $10^{16} \mathrm{GeV}$ scales for small warped extra dimension models [11, or at the lower TeV scales for large flat extra dimension models 12, they (the ADD and RS brane models) also address the long standing puzzle of the gauge hierarchy problem, i.e. the huge discrepancy between the gravitational scale and the electroweak scale. Furthermore, there is a viable Kaluza-Klein WIMP candidate for the dark matter of the universe [13. In addition, extra dimensional models explain the large mass hierarchy of the different types and generations of the SM fermions through a geometrical mechanism. In both, the ADD models 14-17] and in the RS models [18, 19, the SM fermions have different localizations along extra dimension(s) which depend on the type and the flavour of the fermions. This mechanism does not rely on the presence of any new symmetry in the short-distance theory, as in the case of the conventional Froggatt-Nielsen mechanism, which introduces a 'flavor symmetry' 20].

One finds in the literature many higher-dimensional models which have been proposed for generating the SM fermion mass hierarchy [21]. E.g. several frameworks explain the lightness of neutrinos relatively to other SM fermions within the ADD [22, the RS 223, 24] or RS extensions 25.

We concentrate on a RS model, which in contrast with the ADD, does not need of any new energy scale in the fundamental theory. In this RS model, the SM fermion mass hierarchy arises mainly from the dependence on the location, within the warped geometry, of each (type and family) fermion. However, to obtain the correct quark and lepton (masses and) mixing, one must have appropriate Yukawa coupling structures, because, even if the Yukawa couplings do not induce the main features of the fermion mass hierarchy (coming thus from the geometrical mechanism), the structure of the Yukawa texture is still of crucial significance, especially for the mixing. Within the context of the different SM fermion locations along a warped extra dimension, diverse cases have been studied [26], e.g. models with Majorana neutrinos [27, or models with Dirac neutrinos where the charged lepton Yukawa couplings are assumed to be diagonal [28, or models with small Yukawa fluctuations 29, 30. In all these cases, however, the Yukawa couplings did not come from any principle or symmetry, but were chosen in such a way as to merely justify the data.

In this paper, we consider Dirac fermions and give two specific structures for the fermion Yukawa coupling texture, which seem to be particularly appropriate for the RS model, and which account for the experimental data on masses and mixings of all fermions, leptons and quarks. We focus on a permutation symmetry structure and on the Universal Strength for Yukawa couplings (USY) 31. In USY, all Yukawa couplings have equal strength and the difference in the Yukawa structure lies in some complex phase. In both scenarios, all Yukawa couplings are of the same order of magnitude. The main features of the fermion mass hierarchy comes thus from the geometrical mechanism, but the correct mixings and masses are obtained from Yukawa textures which have a definite structure (USY) or a (permutation) symmetry .

In Section 2, we describe the RS model and the geometrical mechanism, which allows for different localizations along the warped extra dimension, together with the resulting fermion mass matrices in 4D. In Section 3, we make a short review of the experimental constraints on the RS

\footnotetext{
${ }^{2}$ See also 32 for extensions of the RS model.
} 
scenario parameters. In Section 4, we concentrate on the structure of the Yukawa couplings and their importance for the mixing. Two appropriate structures for the Yukawa couplings in the RS scenario are introduced: a permutation symmetry and USY. Then, in Section 5 predictions are given for fermion masses and mixing angles based on the two proposed texture scenarios. Finally, we conclude in Section 6 .

\section{Theoretical framework}

\subsection{The RS geometrical scenario}

The RS scenario consists of a 5-dimensional theory, where the extra spatial dimension, parameterized by $y$, is compactified on a $S^{1} / \mathbb{Z}_{2}$ orbifold of radius $R$, such that $-\pi R \leq y \leq \pi R$. Gravity propagates in the bulk and the extra spatial dimension is bordered by two 3-branes with tensions $\Lambda_{y=0}$ and $\Lambda_{y=\pi R}$ (vacuum energy densities) tuned in such way that,

$$
\Lambda_{(y=0)}=-\Lambda_{\left(y=\pi R_{c}\right)}=-\Lambda / k=24 k M_{5}^{3},
$$

where $\Lambda$ is the bulk cosmological constant, $M_{5}$ the fundamental 5-dimensional gravity scale and $k$ a characteristic energy scale (typically of the order $M_{P l}$, ). One finds a solution to the 5-dimensional Einstein's equations which respects 4-dimensional Poincaré invariance. This solution corresponds to a zero mode of the graviton localized on the positive tension brane (the 3 -brane at $y=0$ ) and the non-factorizable metric,

$$
d s^{2}=e^{-2 \sigma(y)} \eta_{\mu \nu} d x^{\mu} d x^{\nu}+d y^{2}, \text { with } \sigma(y)=k|y|,
$$

where $x^{\mu}$ denotes the coordinates of the familiar 4 dimensions and $\eta_{\mu \nu}=\operatorname{diag}(-1,1,1,1)$ the flat metric. The bulk geometry associated to the metric in Eq. (2) is a slice of Anti-de-Sitter $\left(A d S_{5}\right)$ space with curvature radius $1 / k$.

From the fluctuations on the metric of Eq. (2), one derives (integrating over $y$ ) an expression for the effective 4-dimensional gravity scale as a function of the three fundamental RS parameters $k, R$ and $M_{5}$ :

$$
M_{P l}^{2}=\frac{M_{5}^{3}}{k}\left(1-e^{-2 \pi k R}\right) .
$$

On the Planck-brane (the 3-brane at $y=0$ ), the gravity scale is equal to the (reduced) Planck mass: $M_{P l}=1 / \sqrt{8 \pi G_{N}}=2.4410^{18} \mathrm{GeV}\left(G_{N} \equiv\right.$ Newton's constant), while on the TeV-brane (the 3 -brane at $y=\pi R$ ), the gravity scale is affected by the exponential "warp" factor $w=e^{-\pi k R_{c}}$ and becomes much smaller:

$$
M_{\star}=w M_{P l},
$$

Clearly, for a small extra dimension with radius $R \simeq 11 / k$, with $k$ of order $M_{P l}$, one has $w \sim 10^{-15}$ and thus $M_{\star}=\mathcal{O}(1) \mathrm{TeV}$. Hence, the gravity scale $M_{\star}$ on the TeV-brane can be of the same order of magnitude as the electroweak symmetry breaking scale.

In addition, on finds a solution for the gauge hierarchy problem: if the SM Higgs boson is confined on the TeV-brane, it feels a cut-off at $M_{\star}=\mathcal{O}(1) \mathrm{TeV}$ which guarantees the stability of Higgs mass with respect to divergent quantum corrections.

\subsection{The fermion mass matrices in $4 \mathrm{D}$}

In this RS scenario, the SM fermion mass hierarchy is generated through a geometrical mechanism: the fermions reside in the bulk 3 and the SM (zero mode) fermions are given different localizations along the warped extra dimension. Each type of SM fermion field $\Psi_{i}(i$ is the flavor index $)$ is coupled to a mass $m_{i}$ in the 5 -dimensional fundamental theory:

$$
m_{i} \bar{\Psi}_{i} \Psi_{i}
$$

\footnotetext{
${ }^{3}$ Gauge coupling unification and 5-dimensional gauge invariance require the SM gauge bosons to live in the bulk 33. 34. A Kaluza-Klein WIMP candidate (within the RS model) also requires bulk fermions. See 23, for the behavior of fermions in the bulk.
} 
To have different localizations for the zero mode fermions, one must have a non-trivial dependence of the $m_{i}$ on the fifth dimension, i.e. the $m_{i}$, which could be the vacuum expectation values of some scalar fields, must have a '(multi-)kink' profile [5, 35. An attractive possibility is a parameterization of the masses as [36],

$$
m_{i}=c_{i} \frac{d \sigma(y)}{d y}= \pm c_{i} k
$$

where $\sigma(y)$ is defined in Eq.(2) and the $c_{i}$ are dimensionless parameters. This parameterization is compatible with the $\mathbb{Z}_{2}$ symmetry $(y \rightarrow-y)$ of the $S^{1} / \mathbb{Z}_{2}$ orbifold: the masses are odd under the $\mathbb{Z}_{2}$ transformation. Defining the fermion parity with $\Psi_{ \pm}(-y)= \pm \gamma_{5} \Psi_{ \pm}(y)$, one finds that the product $\bar{\Psi}_{ \pm}^{i} \Psi_{ \pm}^{i}$ is also odd under the $\mathbb{Z}_{2}$ transformations, and thus, the whole mass term in Eq. (5) is even.

The equation of motion for the 5 -dimensional fermion fields $\Psi_{i}$ includes the mass term in Eq. (5) and the $\Psi_{i}$ decompose as,

$$
\Psi_{i}\left(x^{\mu}, y\right)=\frac{1}{\sqrt{2 \pi R_{c}}} \sum_{n=0}^{\infty} \psi_{i}^{(n)}\left(x^{\mu}\right) f_{n}^{i}(y)
$$

where $n$ labels the tower of Kaluza-Klein excitations. The zero mode wave function admits the following solution along the extra dimension [18, 23, with normalization factor $N_{0}^{i}$ :

$$
f_{0}^{i}(y)=\frac{e^{\left(2-c_{i}\right) \sigma(y)}}{N_{0}^{i}} \quad ; \quad N_{0}^{i}=\sqrt{\frac{e^{\pi k R\left(1-2 c_{i}\right)}-1}{2 \pi k R\left(1-2 c_{i}\right)}} .
$$

From Eq.(8) one finds that the zero mode of a fermion is more localized towards the Planck-brane if $c_{i}$ increases. Subsequently, the zero mode of the fermion is more localized towards the TeV brane if $c_{i}$ decreases. We shall see that localization differences are important to generate the fermion mass hierarchies.

The SM fermions acquire a Dirac mass through the coupling to a Higgs field. After spontaneous symmetry breaking, and integrating out the extra dimension one obtains:

$$
\int d^{4} x \int d y \sqrt{G}\left(\lambda_{i j}^{(5)} H \bar{\Psi}_{+i} \Psi_{-j}+\text { h.c. }\right)=\int d^{4} x M_{i j} \bar{\psi}_{L i}^{(0)} \psi_{R j}^{(0)}+\text { h.c. }+\ldots,
$$

where $G=e^{-8 \sigma(y)}$ is the determinant of the RS metric. The $\lambda_{i j}^{(5)}$ are the 5-dimensional Yukawa coupling constants and the dots stand for the mass terms of the Kaluza-Klein (KK) excited modes. The effective 4-dimensional mass matrix is given by the integral:

$$
M_{i j}=\int d y \sqrt{G} \frac{\lambda_{i j}^{(5)}}{2 \pi R} H(y) f_{0}^{i}(y) f_{0}^{j}(y) .
$$

Following the motivation from the equation of motion for a bulk scalar field [37, we assume an exponential form for the Higgs field:

$$
H(y)=H_{0} e^{4 k(|y|-\pi R)},
$$

which is shape-peaked at the TeV-brane. As mentioned in Section 2.1 this is a crucial ingredient in obtaining a solution for the gauge hierarchy problem. The amplitude $H_{0}$ can be expressed in terms of the $W^{ \pm}$boson mass, $k R$ and the 5-dimensional weak gauge coupling constant $g^{(5)}$.

After integration of Eq. (10), one obtains an analytical expression for the fermion mass matrix,

$$
M_{i j}=\lambda_{i j} M_{k R}\left(\frac{e^{\pi k R\left(4-c_{i}-c_{j}\right)}-1}{4-c_{i}-c_{j}}\right)\left(\frac{\left(1-2 c_{i}\right)\left(1-2 c_{j}\right)}{\left(e^{\pi k R\left(1-2 c_{i}\right)}-1\right)\left(e^{\pi k R\left(1-2 c_{j}\right)}-1\right)}\right)^{\frac{1}{2}} .
$$

where

$$
M_{k R}=\frac{1}{2} M_{W} \sqrt{\frac{6 \pi k R}{e^{6 \pi k R}-1}}
$$


It is assumed that $\lambda_{i j}^{(5)}=\lambda_{i j} g^{(5)}$. Consequently, there is no $g^{(5)}$ dependence in the expression of Eq. (12), which is exactly compensated by the $g^{(5)}$ dependence in the amplitude $H_{0}$. For each type of fermion, one obtains a different (Dirac) mass matrix ,

$$
M_{i j}^{a}=M_{i j}^{a}\left(k R, \lambda_{i j}^{a}, c_{i}^{A}, c_{j}^{a}\right)
$$

In this notation, $a=l, \nu$ together with $A=L$ for the leptons, while for the quarks, $a=u, d$ with

$A=Q$. The $c_{i}^{L}$ parameterize the 5-dimensional masses of the $S U(2)_{L}$ doublets of the leptons while the $c_{j}^{l}, c_{j}^{\nu}$ parameterize the 5 -dimensional masses of the right handed charged leptons or neutrinos. The $c_{i}^{Q}$ and $c_{j}^{u}, c_{j}^{d}$ apply to the quarks. For each type of fermion we have different Yukawa couplings: $\lambda_{i j}^{l}, \lambda_{i j}^{\nu}$ for the leptons and $\lambda_{i j}^{u}, \lambda_{i j}^{d}$ for the quarks.

From the exponential character of the functions in the expression of $M_{i j}$ in Eq.(12), it is clear that the fermion masses can differ greatly (spanning several orders of magnitude) for each flavor $i, j$, depending on the values of $c_{i}, c_{j}$. This dependence resulted from the overlap between Higgs profile $H(y)$ and zero mode fermion wave function $f_{0}^{i, j}(y)$ which also varies with flavor through the $c_{i}, c_{j}$ parameters (see Eq.(8).

\section{Experimental constraints and Parameter space}

Next, we describe several constraints on the parameters of the model. The parameter space includes the scales $k, M_{5}$ and the radius of the extra dimension $R$, together with the $c_{i}^{L}, c_{j}^{l}, c_{j}^{\nu}$ for the leptons and $c_{i}^{Q}, c_{j}^{u}, c_{j}^{d}$ for the quarks, which parametrize the 5 -dimensional masses.

The bulk curvature must be small compared to the higher-dimensional gravity scale, i.e. $k \leq M_{5}$ if one is to trust the RS solution for the metric (c.f. Eq.(2)) 9]. For the limiting situation (as in [28, 38, 39]) where $k=M_{5}$, there is just one energy scale and $M_{5}=M_{P l}$. This results from Eq.(33) and the fact that one must have $k R \simeq 11$, to have a sufficiently small wrap factor for gravity to be of the order of the $\mathrm{TeV}$ scale on the $\mathrm{TeV}$ brane. We shall keep $k=\mathcal{O}\left(M_{5}\right)$. Indeed, for $k R=10.83$, consistent with a 5 -dimensional gravity scale (on the TeV-brane) of $M_{\star}=4 \mathrm{TeV}$ (c.f. Eq.(4)) and in agreement with the solution proposed for the gauge hierarchy problem, a typical mass value for $m_{K K}$ is obtained of $m_{K K}=1 \mathrm{TeV}$ if one chooses $k=0.1 M_{P l}$. Once the $k$ and $R$ parameter values are known, the $M_{5}$ value is fixed by Eq.(33). For these $k$ and $R$ values, $M_{5}=1.1310^{18} \mathrm{GeV}$. Thus, the two values of the fundamental energy scales $k$ and $M_{5}$ in the RS model are quite close. However from Eq.(3), it is clear that any choice of $k$ such that $0.1 M_{P l}<k<10 M_{P l}$ results in fundamental energy scales $k$ and $M_{5}$ which are close. This enables us to choose other $k R$ values (keeping $k R \simeq 11$ ). Here, we do not focus on the precise value of $k R$. Indeed, slightly different $c_{i}$ parameters can be found, in agreement with the permutation and USY setup of this paper, if one cares to choose other $k R$ or $m_{K K}$ values.

Precision electroweak (EW) data constrain the RS model [30] [40-45]. All fields have KK excited states and these lead to new contributions to physical quantities [46]. Here, we name a few. E.g. the mixing between the top quark and its KK excited states contributes to the $\rho$ parameter, which might exceed the bound set by precision EW measurements 40, 41. However, if one chooses certain localization configurations for quark fields (i.e. certain values for $c_{i}$ quark parameters), some of these problems may be circumvented [30].

The mixing between the EW gauge bosons and their KK modes also induce deviations for some precision EW observables. These go typically as $\left(m_{W} / m_{K K}\right)^{2}$, where $m_{K K}=m_{K K}^{(1)}\left(W^{ \pm}\right)$is the mass of first KK excitation of the $W$ gauge boson 5 . Deviations to the weak gauge boson masses and the $\mathrm{W}$ boson coupling to fermions on the Planck-brane (TeV-brane) lead to experimental bounds on $m_{K K}$. These depend on the localization of the SM fermion fields in the bulk (i.e. the values of mass parameters $c_{i}$ for SM fermions). Typically, one finds $m_{K K} \gtrsim 4 \mathrm{TeV}$ [42, but lower values, down to $m_{K K}=1 \mathrm{TeV}$, are allowed for certain $c_{i}$. A global analysis of these constraints can be found in 29,30 .

Experimental bounds on Flavor Changing (FC) processes constrain the RS model, since significant FC effects can be generated with bulk SM fields [28, 39, 47]. The exchange of heavy lepton

\footnotetext{
${ }^{4}$ The mass of first gauge boson KK excitation is given by $m_{K K}=2.45 k e^{-\pi k R_{c}}$ in the RS model [44].

${ }^{5}$ The difference between $m_{K K}^{(1)}\left(W^{ \pm}\right)$and $m_{K K}^{(1)}\left(Z^{0}\right)$ is insignificant in the RS model.
} 
KK excitations leads to deviations from unitarity for the leptonic mixing matrix, which contributes to FC processes like lepton decays: $\mu \rightarrow e \gamma, \tau \rightarrow \mu \gamma$ and $\tau \rightarrow e \gamma$. In [30] we showed that, even for a small value such as $m_{K K}=1 \mathrm{TeV}$, there exist suitable values for the mass parameters $c_{i}$ for which the branching ratios for these three rare decays are below their experimental upper limit.

With regard to the values of the $c_{i}$ parameters, we assume $\left|c_{i}\right| \approx \mathcal{O}(1)$. Thus, the natural values of 5-dimensional masses $m_{i}$ (c.f. Eq.(6) $)$ appearing in the original action are of the same order of magnitude as the fundamental scale of the RS model, namely the bulk gravity scale $M_{5}$, and we avoid the introduction of new energy scales in the theory. For $k=\mathcal{O}\left(M_{5}\right)$, the absolute values of the $c_{i}$ parameters should be of the order of unity. Typically, here we take,

$$
\left|c_{i}\right| \lesssim 5 \text {. }
$$

A precise analysis on the possible values and restrictions on $m_{K K}$ and the allowed values for the $c_{i}$ is outside the scope of this paper. The $c_{i}$ values (or the exact $k R$ value) that we shall use in this paper are not screened for their ElectroWeak precision compatibility, as we merely wish to illustrate that it is possible, in a first anylisis, to incorporate the data on masses and mixings. We refer to [29, 30], for more details on these, as well as many other effects caused by the diverse KK states.

\section{Yukawa couplings and mass matrices}

\subsection{The structure of the mass matrices}

Next, we give an analysis of the fermion mass matrices $M_{i j}^{a}$ which result from our RS scenario. From the expression for the $M_{i j}$ given in Eq.(13), we find that, to a good approximation, the $M_{i j}$ can be written as

$$
M_{i j}^{a}=g_{i}\left(c_{i}^{A}\right) \cdot \kappa_{i j}^{a} \cdot \widehat{g}_{j}\left(c_{j}^{a}\right)
$$

This splitting of $M_{i j}$ is valid in large regions of the parameter space spanned by $c_{i}^{A}, c_{j}^{a}$. The $g_{i}, \widehat{g}_{j}$ are suitable functions for certain regions. E.g. for the region $1 / 2<c_{i}^{A}, c_{i}^{a}<3 / 4$, the functions are equal, $g_{i}=\widehat{g}_{j}=g$, with

$$
g(x)=\lambda \sqrt{\frac{(2 x-1)}{2-x}} e^{\pi k R_{c}(2-x)} .
$$

where $\lambda$ is some parameter dependent on $k R$ and $M_{W}$ (but irrelevant for the following).

The splitting-structure of $M_{i j}$, however, has important consequences. To see this, take as an example all $\kappa_{i j}=1$. Then, from the structure in Eq. (15), we obtain, e.g. for the neutrinos and the charged leptons, the following expressions for the mass matrices:

$$
\begin{aligned}
& M_{\nu}=D_{L} \cdot \Delta \cdot D_{\nu} \\
& M_{l}=D_{L} \cdot \Delta \cdot D_{l}
\end{aligned} \quad ; \quad \Delta=\left[\begin{array}{ccc}
1 & 1 & 1 \\
1 & 1 & 1 \\
1 & 1 & 1
\end{array}\right]
$$

where $D_{L, l, \nu}=\operatorname{diag}\left(a_{1}, a_{2}, a_{3}\right)_{L, l, \nu}$, and the $a$ 's are obtained from the $g_{i}$ and $\widehat{g}_{j}$ functions in Eq. (15). In this approximation, only the tau and one neutrino eigenstate have mass. However, the point is, that the resulting squared matrices $H_{\nu}=M_{\nu} M_{\nu}^{\dagger}$ and $H_{l}=M_{l} M_{l}^{\dagger}$ are of the same form

$$
\begin{aligned}
H_{\nu} & =\rho_{\nu} D_{L} \cdot \Delta \cdot D_{L} & & \rho_{\nu}=\sqrt{a_{\nu_{1}}^{2}+a_{\nu_{2}}^{2}+a_{\nu_{3}}^{2}} \\
H_{l} & =\rho_{l} D_{L} \cdot \Delta \cdot D_{L} & & \rho_{l}=\sqrt{a_{l_{1}}^{2}+a_{l_{2}}^{2}+a_{l_{3}}^{2}}
\end{aligned}
$$

Thus, the matrices $V_{\nu}$ and $V_{l}$, which diagonalize respectively $H_{\nu}$ and $H_{l}$, are equal; there is no mixing: $V_{M N S}=V_{l}^{\dagger} V_{\nu}=\mathbb{I}$, and although small deviations from $\kappa_{i j}^{l, \nu}=1$ may be sufficient to generate the masses of all other charged leptons and neutrinos, this scenario only leads to small deviations from $V_{M N S}=\mathbb{I}$, for the mixing.

It is clear, in RS scenarios, even if the Yukawa couplings do not induce the main features of the fermion mass hierarchy (coming thus from the geometrical mechanism described), the structure of the Yukawa couplings is of crucial significance for the mixing, and at least for the leptons, some of the $\kappa_{i j}^{l, \nu}$ must be very different from each other. 


\subsection{Successful scenarios for the Yukawa structure}

In this subsection, we give two possible structures for the Yukawa couplings, which are compatible with our RS scenario. We focus a permutation symmetry structure and the Universal Strength for Yukawa couplings (USY) texture [31. In both scenarios, all Yukawa couplings are of the same order of magnitude. Thus, the main features of the fermion hierarchies are explained through the RS geometrical mechanism, and not because some Yukawa coupling is extremely small. Explicitly, we choose $1 / 2<\left|k_{i j}\right|<2$.

\section{(A) Permutations}

Imposing the following permutation structure on the fermion fields:

$$
\begin{array}{cc}
\Psi_{1}^{A} \longrightarrow \Psi_{2}^{A} ; \quad \Psi_{2}^{A} \longrightarrow \Psi_{3}^{A} ; \quad \Psi_{3}^{A} \longrightarrow \Psi_{1}^{A} \\
\Psi_{1}^{a} \longrightarrow \Psi_{2}^{a} ; \quad \Psi_{2}^{a} \longrightarrow \Psi_{3}^{a} ; \quad \Psi_{3}^{a} \longrightarrow \Psi_{1}^{a}
\end{array}
$$

(remember the notation: $a=l, \nu$ when $A=L$ for the leptons, while for the quarks, $a=u, d$ with $A=Q)$. Then, all Yukawa couplings have the following structure:

$$
k=\left[\begin{array}{ccc}
1 & A & B \\
B & 1 & A \\
A & B & 1
\end{array}\right]
$$

In addition to this, the $c_{i}$ 's and $m_{i}$ 's in Eq. (6) of each type of field would be equal. However, at this point, we break the symmetry by some mechanism, e.g. by having different vacuum expectation values for the scalar fields which give rise to the $m_{i}$. Thus, we take $c_{i}^{A} \neq c_{j}^{A}$ and $c_{i}^{a} \neq c_{j}^{a}$ for $i \neq j$. It is known that in the SM, Yukawa couplings of the type in Eq. (20) lead to masses and mixings which are non realistic.

\section{(B) USY}

Another possible structure for the Yukawa couplings is the so called Universal Strength for Yukawa couplings. In USY, all Yukawa couplings have equal strength, $\left|k_{i j}\right|=1$. The difference in the Yukawa structure lies in some complex phase:

$$
k_{i j}=\left[e^{\theta_{i j}}\right]
$$

We shall see that this structure is particularly useful for the neutrinos, as a minimum of two (indeed, curious) complex phases is already sufficient to generate the known neutrino mass differences.

\section{$5 \quad$ Results on fermion masses and mixing}

\subsubsection{The experimental data}

Next, we present the data on fermion masses and mixings. In principle, we consider running masses at the cutoff energy scale of the effective 4 -dimensional theory, i.e. the $\mathrm{TeV}$ range. This scale is also of the order of the electroweak symmetry breaking scale. The predictions for the fermion masses are fitted with the experimental mass values taken at the pole [50. We allow for an uncertainty of $5 \%$ in order to take into account the effect of the renormalization group from the pole mass scale up to the TeV cutoff scale. This is only a few percent 15. In accordance with this uncertainty, we do not determine experimental values with high accuracy. E.g. we take the experimental data on neutrino masses and leptonic mixing angles at the $4 \sigma$ level [49].

With respect to the neutrinos, a general three-flavor fit to the current world's global neutrino data sample has been performed in [49], which includes the results from solar, atmospheric, reactor (KamLAND and CHOOZ) and accelerator $(\mathrm{K} 2 \mathrm{~K})$ experiments. The values for oscillation parameters obtained in this analysis at the $4 \sigma$ level are contained in the intervals:

$$
\begin{aligned}
& 6.8 \leq \Delta m_{21}^{2} \leq 9.3 \quad\left[10^{-5} \mathrm{eV}^{2}\right] \\
& 1.1 \leq \Delta m_{31}^{2} \leq 3.7 \quad\left[10^{-3} \mathrm{eV}^{2}\right]
\end{aligned}
$$


where $\Delta m_{21}^{2} \equiv m_{\nu_{2}}^{2}-m_{\nu_{1}}^{2}$ and $\Delta m_{31}^{2} \equiv m_{\nu_{3}}^{2}-m_{\nu_{1}}^{2}$ are the differences of squared neutrino mass eigenvalues, and,

$$
\begin{gathered}
0.21 \leq \sin ^{2} \theta_{12} \leq 0.41, \\
0.30 \leq \sin ^{2} \theta_{23} \leq 0.72, \\
\sin ^{2} \theta_{13} \leq 0.073,
\end{gathered}
$$

where $\theta_{12}, \theta_{23}$ and $\theta_{13}$ are the three mixing angles from the standard parameterization of the leptonic mixing matrix. The data on tritium beta decay [52] provided by the Mainz and Troitsk 53] experiments give some experimental limits on the effective neutrino mass at $95 \%$ C.L.,

$$
\begin{aligned}
& m_{\beta} \leq 2.2 \mathrm{eV} \quad \text { [Mainz] }, \\
& m_{\beta} \leq 2.5 \mathrm{eV} \quad \text { [Troitsk] }
\end{aligned}
$$

with $m_{\beta}$ defined by, $m_{\beta}^{2}=\sum_{i=1}^{3}\left|V_{e i}\right|^{2} m_{\nu_{i}}^{2}$, where $V_{e i}$ is leptonic mixing matrix..

At $M_{Z}$, the renormalized charged lepton masses are [51]

$$
\begin{aligned}
& m_{e}=0.48684727 \pm 1.410^{-7} \mathrm{MeV} \\
& m_{\mu}=102.75138 \pm 3.310^{-4} \mathrm{MeV} \\
& m_{\tau}=1.74669_{-0.00027}^{+0.00030} \mathrm{GeV}
\end{aligned}
$$

The quark masses and CKM matrix parameters at $M_{Z}$ are give by [51,

$$
\begin{array}{ll}
m_{d}=4.69_{-0.66}^{+0.60} \mathrm{MeV} ; & m_{u}=2.33_{-0.45}^{+0.42} \mathrm{MeV} \\
m_{s}=93.4_{-13.0}^{+11.8} \mathrm{MeV} ; & m_{c}=677_{-61}^{+56} \mathrm{MeV} \\
m_{b}=3.00 \pm 0.11 \mathrm{GeV} ; & m_{t}=181 \pm 13 \mathrm{GeV}
\end{array}
$$

and by

$$
\begin{aligned}
\left|V_{u s}\right| & =0.2205 \pm 0.0018 \\
\left|V_{c b}\right| & =0.0373 \pm 0.0018 \\
\left|V_{u b} / V_{c b}\right| & =0.08 \pm 0.02 .
\end{aligned}
$$

\subsection{Results for two Yukawa scenarios}

Next, we give 4 examples of the parameter values for the $c_{i}$ 's and Yukawa couplings for the two scenarios (permutations and USY), which together with the RS mechanism described, reproduce the known experimental data on fermion masses and mixings.

(A) Permutations

Taking the following values for the $c_{i}^{L}, c_{j}^{l}, c_{j}^{\nu}$ and the permutation matrix parameters $A^{l, v}, B^{l, v}$ in Eq. (20)

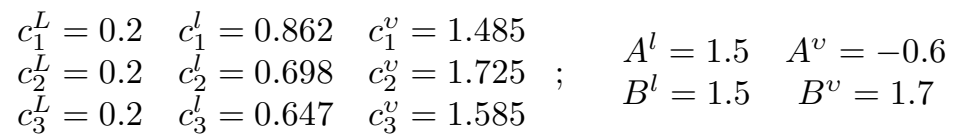

one obtains the leptonic observables,

$$
\begin{aligned}
& m_{e}=0.50 \mathrm{MeV} \quad \Delta m_{21}^{2}=8.8 \quad 10^{-5} \mathrm{eV}^{2} \quad \sin ^{2}\left(\theta_{12}\right)=0.29 \\
& m_{\mu}=103.9 \mathrm{MeV} ; \quad \Delta m_{31}^{2}=3.1 \quad 10^{-3} \mathrm{eV}^{2} ; \quad \sin ^{2}\left(\theta_{23}\right)=0.49 \\
& m_{\tau}=1.74 \mathrm{GeV} \quad m_{v 3}=0.047 \mathrm{eV} \quad \sin ^{2}\left(\theta_{13}\right)=0.016
\end{aligned}
$$

For the quarks, choosing for the $c_{i}^{Q}, c_{j}^{u}, c_{j}^{d}$ and $A^{u, d}, B^{u, d}$

$$
\begin{aligned}
& c_{1}^{Q}=0.100 \quad c_{1}^{u}=0.365 \quad c_{1}^{d}=0.619 \quad A^{u}=1.0072 e^{0.0035 i} \quad A^{d}=1.105 e^{-0.0171 i} \\
& c_{2}^{Q}=0.475 \quad c_{2}^{u}=0.655 \quad c_{2}^{d}=0.652 ; \\
& c_{3}^{Q}=0.100 \quad c_{3}^{u}=0.448 \quad c_{3}^{d}=0.731 \quad B^{u}=1.007 e^{0.0035 i} \quad B^{d}=1.035 e^{-0.0171 i}
\end{aligned}
$$

yields the following $V_{C K M}$ and masses (at $M_{Z}$ ),

$$
\left|V_{C K M}\right|=\left[\begin{array}{lll}
0.9755 & 0.2201 & 0.0036 \\
0.2202 & 0.9747 & 0.0381 \\
0.0081 & 0.0374 & 0.9993
\end{array}\right] ; \quad \begin{array}{llll}
m_{u}=1.1 \mathrm{MeV} & m_{d}=2.7 \mathrm{MeV} \\
& m_{t}=651 & \mathrm{MeV} & m_{s}=83.1 \mathrm{MeV} \\
\mathrm{GeV} & m_{b}=3.1 \mathrm{GeV}
\end{array}
$$


We have allowed for CP violation in the quark sector by choosing just two phases for complex $A^{u, d}, B^{u, d}$ permutation parameters. As a result we obtain for the CP violation parameter $J_{C P} \equiv$ $\left|\operatorname{Im}\left(V_{12} V_{23} V_{22}^{*} V_{13}^{*}\right)\right|=2.810^{-5}$. The experimental values for the angles of the unitarity triangle $\beta \equiv \arg \left(-V_{21} V_{33} V_{23}^{*} V_{31}^{*}\right)$ and $\gamma \equiv \arg \left(-V_{11} V_{23} V_{13}^{*} V_{21}^{*}\right)$, which at this moment measure $\sin (2 \beta)_{\exp }=$ $0.687 \pm 0.032$ and $\gamma_{\exp }=\left(63_{-12}^{+15}\right)^{\circ}$, are near the values found here: $\sin (2 \beta)=0.76$ and $\gamma=73.5^{\circ}$.

(B) USY

In the case of the Universal Strength of Yukawa couplings hypothesis, choosing for the leptons

$$
\begin{array}{lll}
c_{1}^{L}=0.2 & c_{1}^{l}=0.729 & c_{1}^{v}=1.387 \\
c_{2}^{L}=0.2 & c_{2}^{l}=0.637 & c_{2}^{v}=1.423 \\
c_{3}^{L}=0.2 & c_{3}^{l}=0.684 & c_{3}^{v}=1.423
\end{array} \quad ; \quad k^{v}=\left[\begin{array}{ccc}
1 & 1 & 1 \\
1 & -i & 1 \\
1 & 1 & i
\end{array}\right] ; \quad k^{l}=\left[\begin{array}{ccc}
1 & 1 & 1 \\
1 & e^{0.012 i} & 1 \\
1 & 1 & e^{0.563 i}
\end{array}\right]
$$

yields the leptonic observables,

$$
\begin{aligned}
& m_{\mu}=104.6 \mathrm{MeV} ; \quad \Delta m_{31}^{2}=2.410^{-3} \mathrm{eV}^{2} \text {; } \\
& m_{\tau}=1.75 \mathrm{GeV} \quad m_{v 3}=0.050 \mathrm{eV} \\
& \begin{array}{l}
\sin ^{2}\left(\theta_{12}\right)=0.25 \\
\sin ^{2}\left(\theta_{23}\right)=0.39 \\
\sin ^{2}\left(\theta_{13}\right)=0.026
\end{array}
\end{aligned}
$$

Note the peculiar phases in the Yukawa structure for the neutrinos. Taking for the quarks,

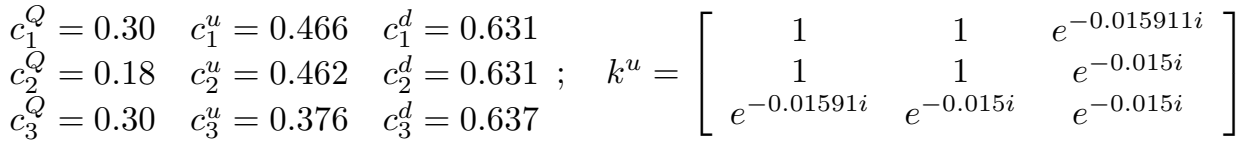

$$
\begin{aligned}
& k^{d}=\operatorname{diag}\left(1, e^{-0.002 i}, e^{-0.045 i}\right) \cdot\left[\begin{array}{ccc}
1 & 1 & e^{0.147 i} \\
1 & 1 & e^{0.175 i} \\
e^{0.147 i} & e^{0.175 i} & e^{0.175 i}
\end{array}\right]
\end{aligned}
$$

results in the following $V_{C K M}$ and masses (at $M_{Z}$ ),

$$
\left|V_{C K M}\right|=\left[\begin{array}{lll}
0.9754 & 0.2202 & 0.0032 \\
0.2201 & 0.9747 & 0.0381 \\
0.0082 & 0.0374 & 0.9993
\end{array}\right] ; \quad \begin{array}{llll}
m_{u}=1.3 & \mathrm{MeV} & m_{d}=2.8 \mathrm{MeV} \\
m_{c}=654 & \mathrm{MeV} & m_{s}=93.1 \mathrm{MeV} \\
m_{t}=186 & \mathrm{GeV} & m_{b}=3.0 \mathrm{GeV}
\end{array}
$$

In the USY case, the Yukawa couplings are already complex. For the quarks and the phases given above, one finds $J_{C P}=2.610^{-5}$ together with $\sin (2 \beta)=0.69$ and $\gamma=76.1^{\circ}$.

\section{Conclusions}

We have introduced, in a RS model with SM fields in the bulk and the Higgs boson on the TeVbrane, two specific structures for the Yukawa couplings, one based on a permutation symmetry and the other on the Universal Strength of Yukawa couplings hypothesis. In both scenarios, all Yukawa couplings are of the same order of magnitude. Thus, the main features of the fermion hierarchies are explained through the RS geometrical mechanism, and not because some Yukawa coupling is extremely small.

We have found that the RS model is particularly appropriate to incorporate the two suggested Yukawa configurations. Indeed, the RS geometrical mechanism of fermion locations along the extra dimension, combined with the two Yukawa scenarios, reproduces all the present experimental data on fermion masses and mixing angles. In particular, we find it remarkable that in the USY case, only two complex phases of definite value $\pm \frac{\pi}{2}$ are sufficient to generate the known neutrino mass differences while at same time permitting large leptonic mixing in agreement with experiment. We also point out that for this RS model, in the USY case for the quarks, the values for the CP violation parameters are much inproved, in contrast with the SM-USY where CP violation is small.

\section{Acknowledgments}

This work was supported by the Fundação para a Ciência e Tecnologia (FCT) of the Portuguese Ministry of Science and Technology, and the EU, through the projects POCTI/FNU/44409/2002 and PDCT/FP/63914/2005. The author is grateful to D. Emmanuel-Costa for useful discussions and to the CERN Physics Theory (PH) Department for the warm hospitality. 


\section{References}

[1] T. Kaluza, Sitzungsber. Preuss. Akad. Wiss. Berlin (Math. Phys.) 1921 (1921) 966; O. Klein, Z. Phys. 37 (1926) 895 [Surveys High Energ. Phys. 5 (1986) 241].

[2] For a review of recent developments, see K. R. Dienes, Phys. Rept. 287 (1997) 447.

[3] T. Appelquist, H.-C. Cheng and B. A. Dobrescu, Phys. Rev. D64 (2001) 035002.

[4] K. Akama, in Gauge Theory and Gravitation, Proceedings of the International Symposium, Nara, Japan, 1982, ed. by K. Kikhawa, N. Nakanishi and H. Nariai (Springer-Verlag, 1983), 267, hep-th/0001113; M. Visser, Phys. Lett. B159 (1985) 22; E. J. Squires, Phys. Lett. B167 (1985) 286; I. Antoniadis, Phys. Lett. B246 (1990) 377.

[5] V. A. Rubakov and M. E. Shaposhnikov, Phys. Lett. B125 (1983) 136.

[6] I. Antoniadis, C. Muñoz and M. Quirós, Nucl. Phys. B397 (1993) 515; I. Antoniadis, K. Benakli and M. Quirós, Phys. Lett. B331 (1994) 313; I. Antoniadis, S. Dimopoulos, A. Pomarol and M. Quirós, Nucl. Phys. B544 (1999) 503.

[7] N. Arkani-Hamed, S. Dimopoulos and G. Dvali, Phys. Lett. B429 (1998) 263; I. Antoniadis, N. Arkani-Hamed, S. Dimopoulos and G. Dvali, Phys. Lett. B436 (1998) 257; N. ArkaniHamed, S. Dimopoulos and G. Dvali, Phys. Rev. D59 (1999) 086004.

[8] M. Gogberashvili, Int. J. Mod. Phys. D11 (2002) 1635.

[9] L. Randall and R. Sundrum, Phys. Rev. Lett. 83 (1999) 3370.

[10] For a pedagogical text, see e.g. D. Bailin and A. Love, "Supersymmetric gauge field theory and string theory", Graduate student series in physics, Institute of physics publishing, ed. by Douglas F. Brewer.

[11] A. Pomarol, Phys. Rev. Lett. 85 (2000) 4004; L. Randall and M. D. Schwartz, JHEP 0111 (2001) 003; L. Randall and M. D. Schwartz, Phys. Rev. Lett. 88 (2002) 081801; W. D. Goldberger and I. Z. Rothstein, Phys. Rev. D68 (2003) 125011; K. Agashe, A. Delgado and R. Sundrum, Annals Phys. 304 (2003) 145.

[12] K. R. Dienes, E. Dudas and T. Gherghetta, Nucl. Phys. B537 (1999) 47; Y. Nomura, D. Smith and N. Weiner, Nucl. Phys. B613 (2001) 147.

[13] E. W. Kolb and R. Slansky, Phys. Lett. B135 (1984) 378; G. Servant and T. M. P. Tait, Nucl. Phys. B650 (2003) 391, and references therein; H.-C. Cheng, J. L. Feng and K. T. Matchev, Phys. Rev. Lett. 89 (2002) 211301; D. Hooper and G. D. Kribs, Phys. Rev. D67 (2003) 055003; K. Agashe and G. Servant, JCAP 0502 (2005) 002 ; K. Agashe and G. Servant, Phys. Rev. Lett. 93 (2004) 231805.

[14] N. Arkani-Hamed and M. Schmaltz, Phys. Rev. D61 (2000) 033005; M. V. Libanov and S. V. Troitsky, Nucl. Phys. B599 (2001) 319; J.-M. Frère, M. V. Libanov and S. V. Troitsky, Phys. Lett. B512 (2001) 169; J.-M. Frère, M. V. Libanov and S. V. Troitsky, JHEP 0111 (2001) 025; M. V. Libanov and E. Ya. Nougaev, JHEP 0204 (2002) 055; G. Dvali and M. Shifman, Phys. Lett. B475 (2000) 295; P. Q. Hung, Phys. Rev. D67 (2003) 095011; D. E. Kaplan and T. M. P. Tait, JHEP 0006 (2000) 020; D. E. Kaplan and T. M. P. Tait, JHEP 0111 (2001) 051; M. Kakizaki and M. Yamaguchi, Prog. Theor. Phys. 107 (2002) 433; Int. J. Mod. Phys. A19 (2004) 1715; C. V. Chang et al., Phys. Lett. B558 (2003) 92; S. Nussinov and R. Shrock, Phys. Lett. B526 (2002) 137.

[15] E. A. Mirabelli and M. Schmaltz, Phys. Rev. D61 (2000) 113011.

[16] G. Barenboim, G. C. Branco, A. de Gouvêa and M. N. Rebelo, Phys. Rev. D64 (2001) 073005; G. C. Branco, A. de Gouvêa and M. N. Rebelo, Phys. Lett. B506 (2001) 115; P. Q. Hung and M. Seco, Nucl. Phys. B653 (2003) 123. 
[17] H. V. Klapdor-Kleingrothaus and U. Sarkar, Phys. Lett. B541 (2002) 332; M. Raidal and A. Strumia, Phys. Lett. B553 (2003) 72; J.-M. Frère, G. Moreau and E. Nezri, Phys. Rev. D69 (2004) 033003.

[18] T. Gherghetta and A. Pomarol, Nucl. Phys. B586 (2000) 141.

[19] D. Dooling and K. Kang, Phys. Lett. B502 (2001) 189.

[20] C. D. Froggatt and H. B. Nielsen, Nucl. Phys. B147 (1979) 277.

[21] K. R. Dienes, E. Dudas and T. Gherghetta, Phys. Lett. B436 (1998) 55; K. R. Dienes, E. Dudas and T. Gherghetta, Nucl. Phys. B537 (1999) 47; K. Yoshioka, Mod. Phys. Lett. A15 (2000) 29; M. Bando, T. Kobayashi, T. Noguchi and K. Yoshioka, Phys. Rev. D63 (2001) 113017; A. Neronov, Phys. Rev. D65 (2002) 044004; N. Arkani-Hamed et al., Phys. Rev. D61 (2000) 116003.

[22] K. R. Dienes, E. Dudas and T. Gherghetta, Nucl. Phys. B557 (1999) 25; N. Arkani-Hamed et al., Phys. Rev. D65 (2002) 024032; N. Arkani-Hamed and S. Dimopoulos, Phys. Rev. D65 (2002) 052003.

[23] Y. Grossman and M. Neubert, Phys. Lett. B474 (2000) 361.

[24] T. Appelquist et al., Phys. Rev. D65 (2002) 105019; T. Gherghetta, Phys. Rev. Lett. 92 (2004) 161601.

[25] G. Moreau, Eur. Phys. J. C40 (2005) 539.

[26] S. Chang, C.S. Kim and M. Yamaguchi, Phys. Rev. D73 (2006) 033002; K.R. Dienes and S. Hossenfelder, Phys. Rev. D74 (2006) 065013; J.D. Lykken, hep-ph/0607149; C. Biggio, F. Feruglio, I. Masina and M. Perez-Victoria, Nucl. Phys. B677 (2004) 451.

[27] S. J. Huber and Q. Shafi, Phys. Lett. B544 (2002) 295; S. J. Huber and Q. Shafi, Phys. Lett. B583 (2004) 293.

[28] S. J. Huber and Q. Shafi, Phys. Lett. B512 (2001) 365.

[29] G. Moreau and J.I. Silva-Marcos, JHEP 0601 (2006) 048.

[30] G. Moreau and J.I. Silva-Marcos, JHEP 0603 (2006) 090.

[31] G.C. Branco, J.I. Silva-Marcos and M.N. Rebelo, Phys. Lett. B237 (1990) 446; Phys. Lett. B428 (1998)136; Phys. Rev. D62 (2000) 073004; Nucl. Phys. B686 (2204) 188; G.C. Branco and J.I. Silva-Marcos, Phys. Lett. B359 (1995) 166; G.C. Branco, D. Emmanuel-Costa, J.I. Silva-Marcos, Phys. Rev. D56 (1997) 107; J.I. Silva-Marcos, Phys .Rev. D59 (1999) 091301;

[32] I. I. Kogan, S. Mouslopoulos, A. Papazoglou, G. G. Ross and J. Santiago, Nucl. Phys. B584 (2000) 313; S. Mouslopoulos and A. Papazoglou, JHEP 0011 (2000) 018; I. I. Kogan, S. Mouslopoulos, A. Papazoglou and G. G. Ross, Nucl. Phys. B595 (2001) 225; N. Kaloper, Phys. Rev. D60 (1999) 123506; I. I. Kogan, S. Mouslopoulos and A. Papazoglou, Phys. Lett. B501 (2001) 140; I. Oda, Phys. Lett. B480 (2000) 305; I. Oda, Phys. Lett. B472 (2000) 59; H. Hatanaka et al., Prog. Theor. Phys. 102 (1999) 1213; C. Csaki and Y. Shirman, Phys. Rev. D61 (2000) 024008; N. Arkani-Hamed, S. Dimopoulos, G. Dvali and N. Kaloper, Phys. Rev. Lett. 84 (2000) 586; R. Gregory, V. A. Rubakov and S. M. Sibiryakov, Phys. Rev. Lett. 84 (2000) 5928; T. Li, Phys. Lett. B478 (2000) 307; L. Randall and R. Sundrum, Phys. Rev. Lett. 83 (1999) 4690; J. Lykken and L. Randall, JHEP 0006 (2000) 014; N. Kaloper, Phys. Lett. B474 (2000) 269; S. Nam, JHEP 0003 (2000) 005; S. Nam, JHEP 0004 (2000) 002.

[33] H. Davoudiasl, J. L. Hewett and T. G. Rizzo, Phys. Lett. B473 (2000) 43; A. Pomarol, Phys. Lett. B486 (2000) 153.

[34] S. Chang et al., Phys. Rev. D62 (2000) 084025 ; B. Bajc and G. Gabadadze, Phys. Lett. B474 (2000) 282. 
[35] R. Jackiw and C. Rebbi, Phys. Rev. D13 (1976) 3398.

[36] A. Kehagias and K. Tamvakis, Phys. Lett. B504 (2001) 38.

[37] W. D. Goldberger and M. B. Wise, Phys. Rev. Lett. 83 (1999) 4922.

[38] S. J. Huber and Q. Shafi, Phys. Lett. B498 (2001) 256.

[39] S. J. Huber, Nucl. Phys. B666 (2003) 269.

[40] J. L. Hewett, F. J. Petriello and T. G. Rizzo, JHEP 0209 (2002) 030.

[41] C. S. Kim, J. D. Kim and J. Song, Phys. Rev. D67 (2003) 015001.

[42] S. J. Huber and Q. Shafi, Phys. Rev. D63 (2001) 045010.

[43] S. J. Huber, C.-A. Lee and Q. Shafi, Phys. Lett. B531 (2002) 112.

[44] H. Davoudiasl, J. L. Hewett and T. G. Rizzo, Phys. Rev. D63 (2001) 075004.

[45] G. Burdman, Phys. Rev. D66 (2002) 076003.

[46] E. De Pree, M. Sher, Phys. Rev. D73 (2006) 095006; K. Agashe, A.E. Blechman and F. Petriello, Phys. Rev. D74 (2006) 053011; K. Agashe, G. Perez and A. Soni, hep-ph/0606293 S. Chang, C.S. Kim and J. Song, hep-ph/0607313; A. Djouadi, G. Moreau and F. Richard, hep-ph/0610173.

[47] F. del Aguila and J. Santiago, Phys. Lett. B493 (2000) 175.

[48] P. Huber et al., Nucl. Phys. B665 (2003) 487.

[49] M. Maltoni, T. Schwetz, M. A. Tortola, J. W. F. Valle, New J. Phys. 6 (2004) 122, hep-ph/0405172.

[50] Particle Data Group, W.-M. Yao et al., J. Phys. G33 (2006) 1.

[51] H. Fusaoka and Y. Koide, Phys. Rev. D57 (1998) 3986.

[52] Y. Farzan, O. L. G. Peres and A. Yu. Smirnov, Nucl. Phys. B612 (2001) 59.

[53] V. M. Lobashev et al., Phys. Lett. B460 (1999) 227; Nucl. Phys. Proc. Suppl. 77 (1999) 327; 91 (2001) 280; J. Bonn et al., Nucl. Phys. Proc. Suppl. 91 (2001) 273. 